\title{
Osteoporosis Pseudoglioma Syndrome
}

National Cancer Institute

\section{Source}

National Cancer Institute. Osteoporosis Pseudoglioma Syndrome. NCI Thesaurus. Code C130998.

An autosomal recessive condition caused by homozygous or compound heterozygous inactivating mutation(s) in the gene LRP5, encoding low-density lipoprotein receptorrelated protein 5 . This condition is characterized by severe juvenile-onset osteoporosis and congenital or juvenile-onset blindness due to a vascularized retinal mass that resembles a glioma. 